\title{
INFLUENCE DU MODE DE SÉCHAGE SUR LA VALEUR ALIMENTAIRE DE LA LEVURE DE DISTILLERIE
}

\author{
PAR \\ R. FEV RIER \\ Station de recherches \\ sur l'Élevage, C. N. R. Z., \\ Jouy-en-Josas \\ A. M. LEROY \\ Laboratoire de Zootechnie, \\ Institut national \\ agronomique, Paris

\section{J. ROCHE} \\ Laboratoire de \\ Biochimie, Collège \\ de France, Paris
}

Deux d'entre nous ont eu l'occasion (6) d'étudier la valeur de la levure de distillerie comme aliment du bétail. A cette époque, nous avions été frappés par la variabilité des aspects sous lesquels se présentaient les échantillons et, depuis lors, l'utilisation courante de ce produit nous a permis de répéter cette observation.

D'une manière générale, ces différences semblent provenir du séchage, effectué selon l'un des trois procédés suivants:

1. - "Atomiseur " (levure X). - L'émulsion de levure est pulvérisée dans un courant d'air préalablement chauffé à $\mathrm{I} 4 \mathrm{O}^{\circ}$. Les gouttelettes tombent rapidement et sont le siège d'une évaporation intense. La levure atomisée se présente sous forme de poudre fine.

2. - Haute température (levure $Y$ - levure $Y^{\prime}$ ). - L'émulsion de levure séjourne sur des cylindres chauffés à la vapeur à $130^{\circ}$ pendant Io secondes environ. Cette levure se présente sous forme de paillettes.

3. - Basse température (levure $Z$ - levure $Z^{\prime}$ ). - La levure est séchée dans un courant d'air à $60^{\circ}$ pendant 30 à 45 minutes, au cours desquelles des raclettes en rotation lente assurent l'agitation nécessaire de la masse. Elle se présente sous forme de granulés.

Pour les industriels comme pour les éleveurs, il est important de savoir si les différences constatées dans la couleur et la texture du produit séché vont de pair avec des différences de sa valeur alimentaire. Aussi avons-nous comparé, à cet égard, des levures séchées selon les diverses méthodes que nous venons d'indiquer ; trois de celles étudiées dans ce travail avaient été cultivées sur les 
jus de betteraves, et deux sur mélasse (" levure de betterave " et " levure de mélasse $)$.

Le porc a été choisi comme animal d'expérience : sa croissance manifeste une grande sensibilité à la qualité de sa ration et, par ailleurs, la levure a pris une importance relativement grande dans son alimentation.

Cette étude a été réalisée dans la porcherie expérimentale récemment construite à Bois-Corbon. 30 porcs y sont logés dans 30 cases de petites dimensions, aménagées selon le type danois (9) où ils peuvent être alimentés individuellement. Les troubles qu'un isolement intégral des animaux est susceptible de provoquer sont évités par le cloisonnement en tubes d'acier réalisé pour délimiter ces cases.

\section{Première expérience - Levures de betterave}

Nous avons utilisé pour cette étude des porcelets Large White Yorkshire, issus du même verrat et constituant un ensemble aussi homogène que possible. Ils pesaient une trentaine de $\mathrm{kg}$ lorsqu'ils furent mis en observation pendant 4 semaines. A la suite de cette période, nous les avons séparés en trois lots de 8 animaux, qui s'étaient comportés de façon identique. Le tableau ci-dessous donne le détail de cette répartition :

\begin{tabular}{lllll} 
Lot & \multicolumn{4}{l}{ No des sujets } \\
$\mathrm{X}$ & 2162 & 2164 & $217 \mathrm{I}$ & 2153 \\
& 2202 & 2198 & 2157 & $218 \mathrm{I}$ \\
$\mathrm{Y}$ & 2206 & 2185 & 2163 & 2193 \\
& 2195 & 2146 & 2182 & 2173 \\
& & & \\
$Z$ & 2175 & 2199 & 2165 & 2203 \\
& 2168 & 2197 & 2154 & 2167
\end{tabular}

Les résultats de nos observations, rapportés à chaque lot, ont été les suivants, en ce qui concerne le poids à la fin de cette période $(\mathrm{P})$, le gain de poids moyen quotidien (G.M) et l'indice de consommation moyen (I.C) observés :

\begin{tabular}{cccc} 
& \multicolumn{3}{c}{ Moyenne par animal } \\
Lot & $\mathrm{P}$ & G.M. & I.C. \\
\hdashline & $-\mathrm{kg}$ & - & - \\
$\mathrm{X}$ & 44,0 & 577 & 3,25 \\
$\mathrm{Y}$ & 44,0 & 583 & 3,22 \\
$\mathrm{Z}$ & $44, \mathrm{I} 2$ & 583 & 3,23 \\
témoin & 42,5 & $6 \mathrm{r} 3$ & 3,00
\end{tabular}

Chacun des 3 lots a reçu par la suite un régime particulier et les six animaux restants ont constitué un lot témoin.

\section{Régimes}

Nous nous sommes proposés de réaliser des régimes aussi semblables que possible, sauf en ce qui concerne le facteur dont nous voulions étudier l'influence : le mode de séchage de la levure.

L'idéal aurait été de partir du même lot de celle-ci et d'en sécher diffé- 
rentes fractions avec les procédés que nous avons décrits. Cette façon de procéder s'est révélée impraticable, en raison de la masse totale de levure sèche nécessaire (voisine de 2 tonnes (1) et des difficultés de transport du produit brut aux séchoirs industriels distants de plusieurs centaines de kilomètres.

La levure de distillerie étant habituellement utilisée comme aliment azoté, nous avons pensé que des différences éventuelles dans son efficacité pourraient provenir de la qualité de ses constituants azotés. C'est pourquoi nous avons préparé des régimes isoazotés et isoénergétiques différant seulement par la présence de levures ayant subi des traitements thermiques divers.

Nous avons employé la levure comme seul aliment azoté ajouté à de la farine d'orge et à un mélange minéral identique pour tous les lots, afin d'éviter de compléter inégalement certaines carences éventuelles en acides aminés. De ce fait, nous avons été amenés à employer des doses très importantes de levure, supérieures aux maxima habituellement utilisés et considérés par BRAUDE et KoN (I-2) comme rachitigènes. Aucun accident osseux n'a pourtant été signalé et la tolérance digestive des divers régimes a été satisfaisante pendant toute la durée des essais. Notons d'ailleurs que MaCraE (ro) a utilisé des taux de levure atteignant $20 \mathrm{p}$. IOO du régime et que GARTNER et GAEDE (5) ont fait ingérer jusqu'à $600 \mathrm{~g}$ de levure de bière par animal et par jour, sans que des signes de rachitisme n'apparaissent.

Le taux de matières azotées a été fixé par comparaison avec la ration distribuée aux animaux témoins. L'aliment standard utilisé a comporté deux formules, l'une convenant aux jeunes porcs (taux de matières azotées totales I60), l'autre aux pores de plus de $60 \mathrm{~kg}$ (taux de matières azotées totales I30). Aussi la composition de la ration expérimentale a-t-elle été modifiée selon cette norme lorsque les animaux ont atteint ce poids.

L'analyse des différents échantillons a donné les résultats suivants :

\begin{tabular}{|c|c|c|c|c|c|c|}
\hline & $\begin{array}{c}\text { M. } \\
\text { sèche }\end{array}$ & $\begin{array}{c}\text { M. } \\
\text { azotées }\end{array}$ & $\begin{array}{c}\text { M. } \\
\text { grasses }\end{array}$ & $\begin{array}{l}\text { M. } \\
\text { cellul. }\end{array}$ & $\begin{array}{c}\text { M. } \\
\text { minérales }\end{array}$ & $\begin{array}{l}\text { Acidité } \\
\left(\mathrm{SO}_{4} \mathrm{H}_{2}\right)\end{array}$ \\
\hline Levure $\mathrm{X}$ & 874 & 304 & $\overline{45}$ & - & $\overline{82}$ & $I 1,9$ \\
\hline & 889 & $440^{\circ}$ & 37 & - & 72 & 5,7 \\
\hline $\mathrm{Z}$ & 884 & 398 & 16 & $一$ & 44 & 0,9 \\
\hline Orge & 888 & 96 & 25 & 68 & 53 & I, 5 \\
\hline
\end{tabular}

2 - Vitamines et acides aminés (2)

Vitamines

Acides aminés (en mmg par $\mathrm{kg}$ de MS) (N de l'acide aminé rapporté à $\mathrm{N}$ protéique $\times$ roo)

\begin{tabular}{|c|c|c|c|c|c|c|c|}
\hline & $\mathrm{B}_{1}$ & $\mathrm{~B}_{2}$ & $\mathrm{PP}$ & Tyrosine & Tryptophane & Méthionine & Lysine \\
\hline evure $\underset{\mathrm{V}}{\mathrm{X}}$ & 15 & I 5 & 74, & 4,75 & $\mathrm{I}, 4$ & $\mathrm{I}, 3$ & Io, 27 \\
\hline & 19 & 29,3 & b & 4,35 & I, I & $\mathrm{I}, 4$ & 9,60 \\
\hline " Z & 18,9 & 7,4 & 69,0 & 5,10 & $\mathrm{I}, 5$ & 1,3 & $10,3^{8}$ \\
\hline
\end{tabular}

(1) Ce qui correspond à 7 tonnes avant séchage.

$\left({ }^{2}\right)$ Nous remercions M. L. Chevillard, Directeur du Laboratoire de Biochimie des Vitamines à: !'École des Hautes-Etudes, d'avoir bien voulu doser diverses vitamines dans les levures étudiées. 
Voici les formules des différentes rations : Croissance (C) - Engraissement (G)

Orge .

Or.................

Levure...$\ldots \ldots \ldots \ldots$.

Mélange minéral $\ldots \ldots \ldots$.

Manioc.............

Farine de viande ..........

Farine de poisson .........

Tourteau d'arachide .......

\begin{tabular}{cccc} 
Lot X & & \multicolumn{2}{c}{ Lot $\mathrm{I}$} \\
\hline $\mathrm{C}$ & $\frac{\mathrm{G}}{\mathrm{C}}$ & - & $\mathrm{G}$ \\
64 & 80 & 77 & 87 \\
33 & $\mathrm{I} 8$ & 20 & II \\
3 & 2 & 3 & - \\
- & - & - & - \\
- & - & - & -
\end{tabular}

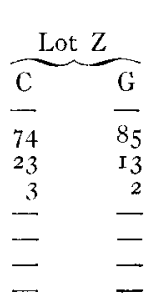

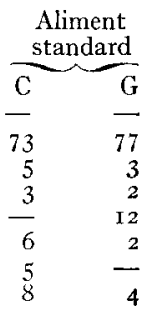

Les rations ont été distribuées en trois repas quotidiens, à 7 - I $2-$ I7 heures, d'après la méthode que nous avons précédemment décrite $(6-7)$. Les animaux recevaient, en outre, $2500 \mathrm{U}$. I. de vitamine $A$ et $250 \mathrm{U}$. I. de vitamine $\mathrm{D}$ sous forme d'huile vitaminée, doses utilisées par les stations danoises (3) et voisines de celles recommandées par le National Research Council (II). Peut-être l'absence des accidents de rachitisme, que BRAUdE et Kon (2) avaient efficacement traités par l'addition d'huile de foie de morue à la ration, est-elle liée à l'ingestion de vitamine D.

\section{Résultats}

Les essais ont duré du 22 novembre I949 au 24 janvier 1950. Les résultats sont présentés dans le tableau ci-dessous :

\begin{tabular}{|c|c|c|c|c|c|}
\hline & $\begin{array}{l}\text { Poids } \\
\text { départ. }\end{array}$ & $\begin{array}{l}\text { Poids } \\
\text { fin. }\end{array}$ & $\begin{array}{c}\text { Consommation } \\
\text { moyenne } \\
\text { quotidienne. }\end{array}$ & $\begin{array}{c}\text { Gain moyen } \\
\text { quotidien. }\end{array}$ & $\begin{array}{c}\text { Indice } \\
\text { moyen de } \\
\text { Consommation. }\end{array}$ \\
\hline Lot $X$ & 49 , & 89,5 & $2,82 \mathrm{~kg}$ & $6_{5} \mathrm{Ig}$ & $4,3^{6}$ \\
\hline & 49,0 & 84,0 & 2,46 & $55^{6}$ & 4,58 \\
\hline & 48,5 & 87,5 & 2,78 & 619 & $4,5 \mathrm{I}$ \\
\hline & 47,7 & 86,7 & 2,73 & & $4,4 \mathrm{I}$ \\
\hline
\end{tabular}

L'étude statistique de ces résultats a permis de calculer les variances relatives à ces trois tests :

\begin{tabular}{|c|c|c|c|}
\hline Variance. & Consommation. & $\begin{array}{l}\text { Gain moyen } \\
\text { quotidien. }\end{array}$ & $\begin{array}{l}\text { Indice de } \\
\text { consommation. }\end{array}$ \\
\hline $\begin{array}{l}\text { Entre lot } \\
\text { Résiduelle }\end{array}$ & $\begin{array}{l}0,33 \\
0,18\end{array}$ & $\begin{array}{r}\text { I } 3,816 \\
\text { I, } 580\end{array}$ & $\begin{array}{l}0,42 \\
0,266\end{array}$ \\
\hline
\end{tabular}

Aucune différence signicative ne peut être relevée entre les trois lots, en ce qui concerne l'indice de consommation et la consommation moyenne quotidienne. Il n'en est pas de même pour les croissances quotidiennes. L,e calcul montre que celle du lot $\mathrm{Y}$ est différente des deux autres qui doivent, statistiquement, être considérées comme équivalentes, malgré la valeur plus élevée du gain de poids moyen enregistré avec l'une d'elles (X).

En utilisant la méthode de calcul indiquée dans un précédent mémoire (4), nous avons pu comparer les indices de consommation, de façon à pouvoir en tirer des conclusions valables sur l'efficacité de la ration. En fixant à $\mathrm{I}$ la valeur de l'efficacité de l'aliment du lot $\mathrm{X}$, nous avons obtenu :

$\begin{array}{ll}\operatorname{lot} X & \mathrm{I} \\ \operatorname{lot} \mathrm{Y} & \mathrm{I}, 02 \\ \operatorname{lot} Z & 0,98\end{array}$


Il est donc légitime de conclure qu'aucune différence appréciable ne peut être relevée entre l'efficacité des trois levures utilisées.

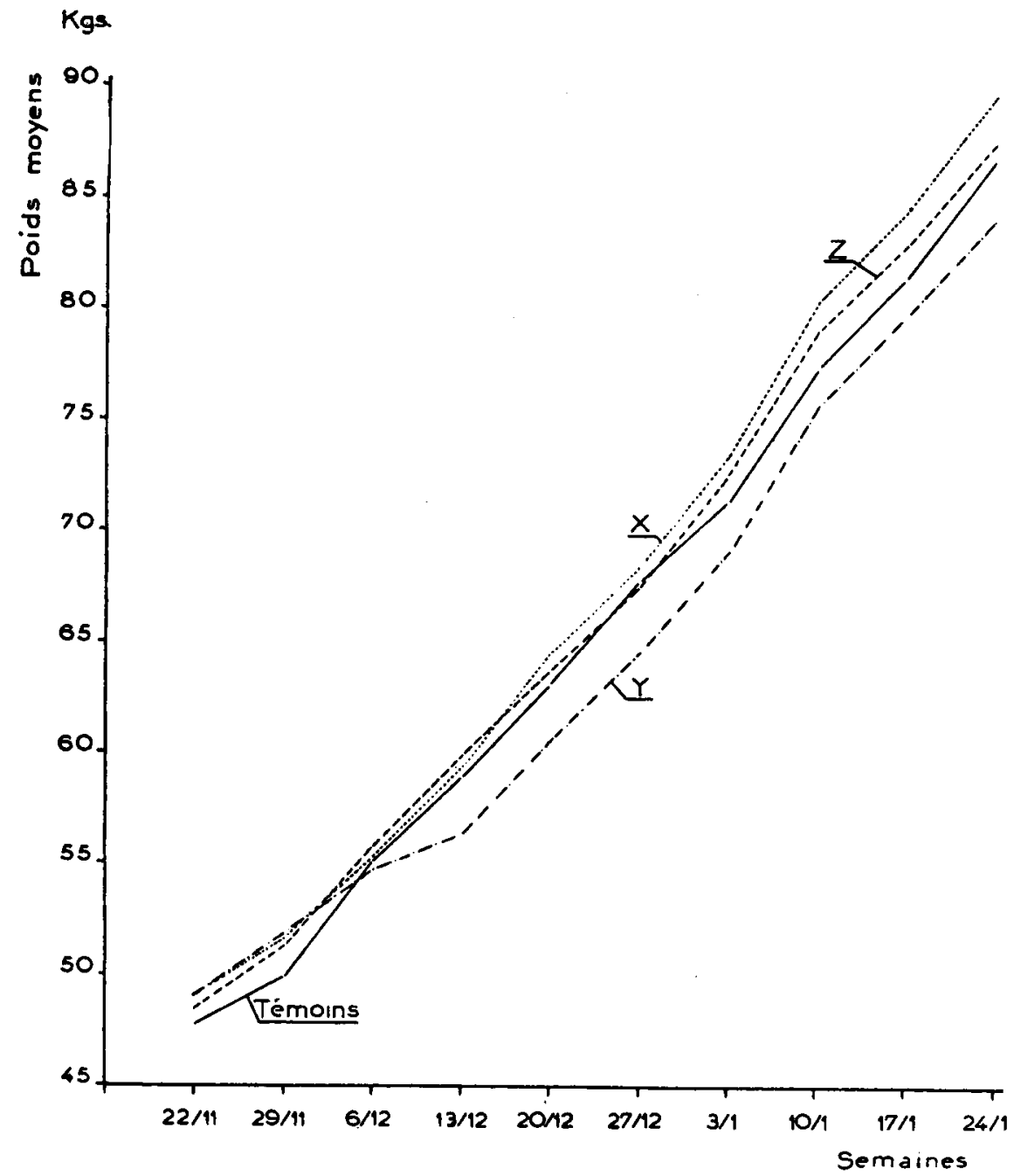

GRAPHIQUE I.

Par contre, nous pouvons dresser le tableau suivant en ce qui concerne la consommation des aliments (valeurs moyennes) :

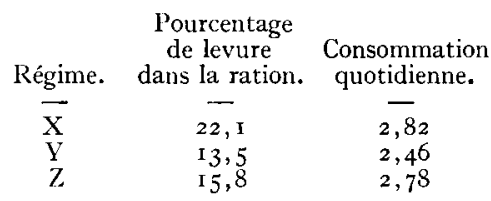


Nous retrouvons ici un phénomène que nous avions déjà observé (6) : à une proportion croissante de levure dans la ration correspond une consommation accrue. Les écarts constatés ne permettent pas d'exprimer mathématiquement cette corrélation, mais sa répétition mérite d'être signalée. Notons d'ailleurs que Tosic (I2) a observé une action favorable d'extraits de levure sur l'appétit du mouton.

Il semble qu'il faille attribuer à la différence d'appétence des rations (provenant de leur teneur plus ou moins grande en levure) les variations que l'on relève dans les effets des régimes étudiés sur la vitesse de croissance et sur l'indice de consommation.

\section{Deuxième expérience - Isevures de mélasse}

Nous disposions de 2I porcelets provenant de l'élevage de Bois-Corbon et issus du même verrat, qui ont été répartis en trois lots ( 2 lots expérimentaux et I lot témoin) en fonction de leur comportement antérieur et de leur origine maternelle :

$\begin{array}{lllll}\text { Lot } \mathrm{Y}^{\prime} & \left\{\begin{array}{l}873 \\ 882\end{array}\right. & 890 & 914 & 898 \\ \text { Lot } Z^{\prime} & \left\{\begin{array}{l}\text { 14 } \\ 869\end{array}\right. & 900 & 93^{2} & \\ 880 & 919 & 934 \\ \text { Lot témoin } & \left\{\begin{array}{l}11 \\ 870\end{array}\right. & 896 & 930 & \\ 887 & 907 & 939 & 88 \mathrm{I}\end{array}$

Ces animaux avaient été étudiés du 25 juillet au I7 août et les observations suivantes avaient été faites :

\begin{tabular}{|c|c|c|c|}
\hline & $\begin{array}{l}\text { Poids moyen } \\
\text { le i } 5 / 8\end{array}$ & $\begin{array}{c}\text { Gain moyen } \\
\text { quotidien } \\
-\end{array}$ & $\begin{array}{l}\text { Indice moyen } \\
\text { de } \\
\text { consommation. }\end{array}$ \\
\hline $\mathrm{l}$ & $\begin{array}{l}\mathrm{kg} \\
\mathbf{2 7}, 0\end{array}$ & $\begin{array}{l}\mathrm{gr} \\
306\end{array}$ & 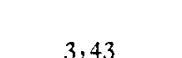 \\
\hline Lot $\mathrm{Y}^{\prime}$ & 26,8 & 299 & $\begin{array}{l}3,4,5 \\
3,46\end{array}$ \\
\hline Iot témoin & 28,4 & 306 & $3,5 \mathrm{I}$ \\
\hline
\end{tabular}

\section{Régimes}

Le problème précédemment évoqué a été plus aisé à résoudre : les deux échantillons provenaient en effet de la même usine où existaient deux séchoirs, l'un travaillant à basse température, l'autre à haute température. Il a donc été plus facile de composer des régimes comparables. Mais les échantillons étant légèrement différents ( $\left.{ }^{1}\right)$ quant à leur teneur en matières azotées, nous avons établi les formules pour obtenir des mélanges isoazotés, d'après les mêmes critères que pour l'expérience précédente.

(1) Lors de la campagne 1946-47, nous avons dosé la teneur en matières azotées d'échantillons prélevés tous les jours. Les résultats montrent que. d'un jour à l'autre, des différences sensibles peuvent être constatées dans les caractéristiques de la levure produite. 
L'analyse avait donné les résultats suivants :

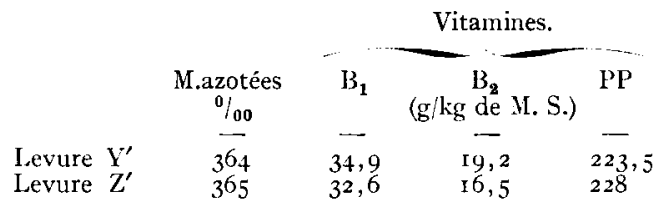

\begin{tabular}{|c|c|c|c|c|c|c|}
\hline \multirow[b]{2}{*}{ Lysine } & \multirow{2}{*}{$\begin{array}{c}\text { Acides amin } \\
\text { Tyrosine }\end{array}$} & (N de l'acide al & nine ra & a prote & ue $\times$ roo) & $\begin{array}{l}\text { Cystine- } \\
\text { cystéine } \\
\text { exprimé en }\end{array}$ \\
\hline & & Tryptophane & Arginine & Histidine & Méthionine & cystine \\
\hline 7 & $\begin{array}{l}3,98 \\
4,10\end{array}$ & $\begin{array}{l}\mathrm{I}, \mathrm{IO} \\
\mathrm{I}, 20\end{array}$ & 4,45 & $\begin{array}{l}\mathrm{I}, 85 \\
2,10\end{array}$ & $\begin{array}{l}\mathrm{I}, 30 \\
\mathrm{I}, \mathrm{IO}\end{array}$ & $\begin{array}{l}0,90 \\
0,85\end{array}$ \\
\hline & 4,10 & $\mathrm{I}, 20$ & 4,25 & & $\mathrm{I}, \mathrm{IO}$ & 0,85 \\
\hline
\end{tabular}

D'autre part, des observations antérieures (6) nous ayant montré que l'influence spécifique de la levure se manifeste essentiellement sur les jeunes sujets, nous avons arrêté nos expériences lorsque le poids moyen du lot le plus lourd a dépassé $60 \mathrm{~kg}$ ( $\mathrm{I} 7$ octobre). Nous n'avons donc pas eu à modifier les formules des régimes en cours d'expérience.

Nous appelons :

$\mathrm{Y}^{\prime}$ le lot comportant de la levure séchée à haute température

$Z^{\prime}$ le lot comportant de la levure séchée à basse température.

Voici les formules des différentes rations :

\begin{tabular}{|c|c|c|c|}
\hline Lot & $\mathrm{Y}^{\prime}$ & $\mathrm{Z}^{\prime}$ & $\begin{array}{l}\text { Aliment } \\
\text { standard. }\end{array}$ \\
\hline Drge $\ldots \ldots \ldots \ldots \ldots \ldots$ & $7 \mathrm{r}$ & 72 & 73 \\
\hline Levure... & 26 & 25 & 5 \\
\hline élange minéral & 3 & 3 & 3 \\
\hline urteau d'arachide .... & $\underline{-}$ & - & 8 \\
\hline arine de viande ....... & - & - & 6 \\
\hline Farine de poisson & - & 一 & 5 \\
\hline
\end{tabular}

Les rations ont été distribuées dans les conditions précisées plus haut.

\section{Résultats}

Ils sont rassemblés dans le tableau suivant:

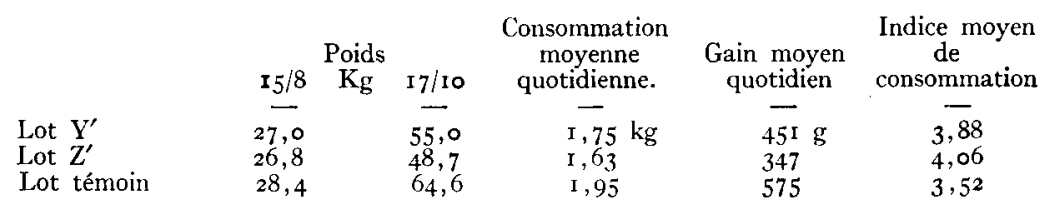

L'étude statistique de ces résultats a permis de calculer les variances relatives à ces trois tests :

\begin{tabular}{|c|c|c|c|}
\hline Vari & $\begin{array}{c}\text { Consommation } \\
\text { moyenne } \\
\text { quotidienne. } \\
-\end{array}$ & $\begin{array}{c}\text { Gain moyen } \\
\text { quotidien. } \\
-\end{array}$ & $\begin{array}{l}\text { Indice de } \\
\text { consommation } \\
\ldots\end{array}$ \\
\hline Ré & $\begin{array}{l}0,12 \\
0,18\end{array}$ & $\begin{array}{r}90, \infty 0 \\
6,14\end{array}$ & $\begin{array}{l}3, \mathrm{I} 3 \\
0, \mathrm{II}\end{array}$ \\
\hline
\end{tabular}

Le calcul montre que les différences sont significatives en ce qui concerne le gain moyen quotidien et les indices de consommation, mais non en ce qui concerne les consommations. 
Les efficacités des divers régimes, en prenant celle du lot témoin pour $I$, sont respectivement :

$\begin{array}{ll}\text { lot témoin } & \mathrm{I} \\ \text { lot } \mathrm{Y}^{\prime} & 0,94 \\ \text { lot } Z^{\prime} & \mathrm{I}\end{array}$

Les levures présentent donc des efficacités voisines, quoique celle de la levure $\mathrm{Y}^{\prime}$ semble légèrement inférieure. La ration du lot témoin a été consommée en plus grande quantité, ce qui permet d'expliquer l'indice de consommation inférieur obtenu avec elle.

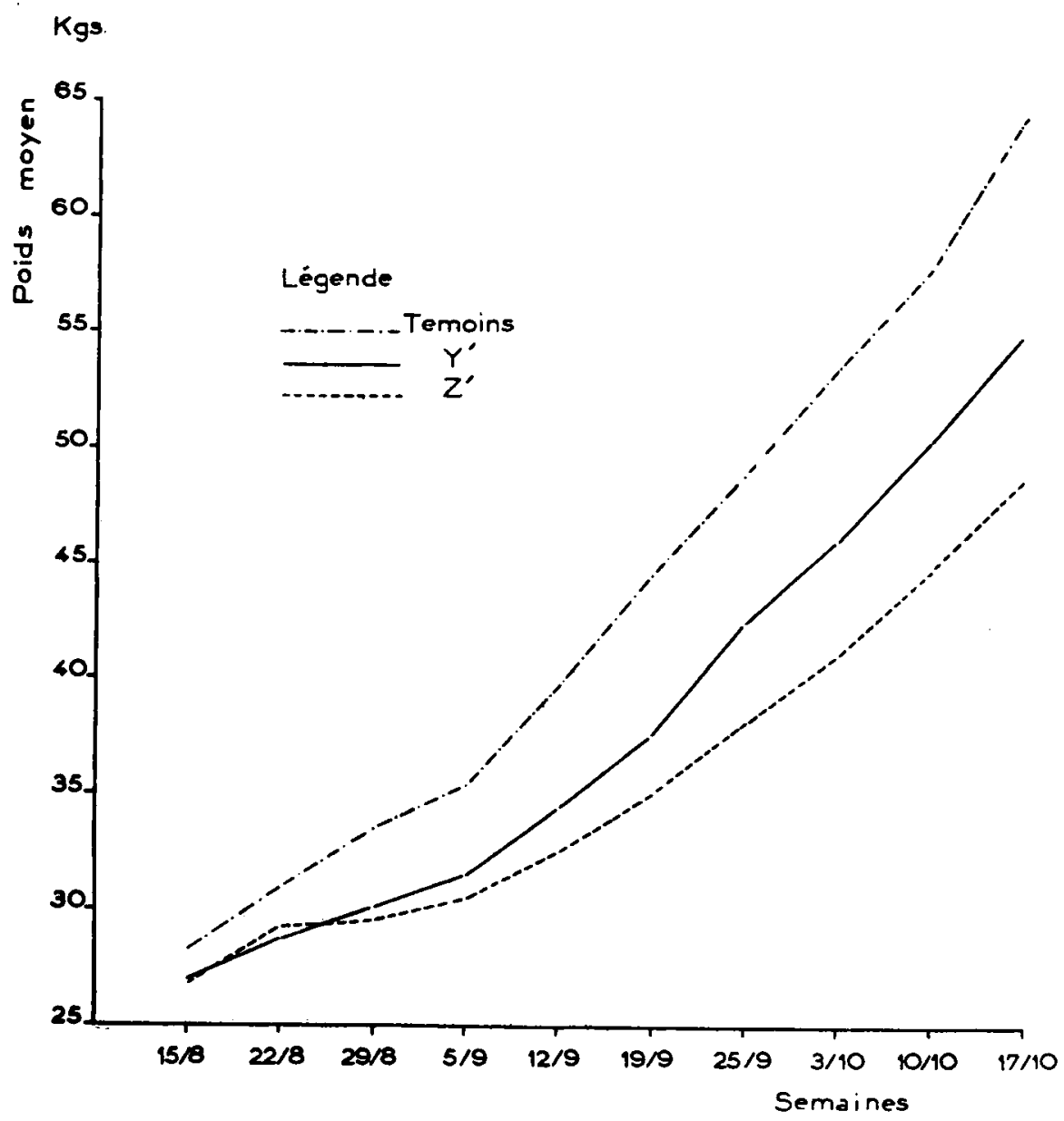

GRAPHIQUE II

Il y a lieu de remarquer la supériorité de l'appétence observée dans le lot témoin, alors que, jusqu'ici, il semblait que les levures amélioraient toujours l'appétence d'une ration. Cette règle ne s'est pas vérifiée pour les deux levures de mélasse et il y aurait lieu d'étendre sur ce point nos observations. 


\section{Examen d'ensemble des résultats et conclusions générales}

Les résultats obtenus se dégagent du tableau suivant:

\begin{tabular}{|c|c|c|c|c|}
\hline & & $\begin{array}{c}\text { Consommation } \\
\text { moyenne } \\
\text { quotidienne. }\end{array}$ & $\begin{array}{l}\text { Gain de poids } \\
\text { moyen } \\
\text { quotidien. }\end{array}$ & $\begin{array}{c}\text { Indice moyen } \\
\text { de } \\
\text { consommation. } \\
-\end{array}$ \\
\hline $\mathrm{I}^{\mathrm{re}}$ expérience & $\left\{\begin{array}{l}\mathrm{X} \\
\mathrm{Y} \\
\mathrm{Z} \\
\text { témoin }\end{array}\right.$ & $\begin{array}{l}\mathrm{kg} \\
2,82 \\
2,46 \\
2,78 \\
2,73\end{array}$ & $\begin{array}{l}\mathrm{g} \\
65 \mathrm{I} \\
556 \\
619 \\
619\end{array}$ & $\begin{array}{l}4,36 \\
4,58 \\
4,51 \\
4,41\end{array}$ \\
\hline $2^{\mathrm{e}}$ expérience & $\left\{\begin{array}{l}\mathrm{Y}^{\prime} \\
\mathrm{Z}^{\prime} \\
\text { témoin }\end{array}\right.$ & $\begin{array}{l}\mathrm{I}, 75 \\
\mathrm{I}, 63 \\
\mathrm{I}, 95\end{array}$ & $\begin{array}{l}451 \\
347 \\
575\end{array}$ & $\begin{array}{l}3,88 \\
4,06 \\
3,52\end{array}$ \\
\hline
\end{tabular}

La comparaison de l'efficacité des levures de betteraves $(X-Y-Z)$ et des levures de mélasse $\left(\mathrm{Y}^{\prime}-\mathrm{Z}^{\prime}\right)$ dans l'alimentation du porc en croissance, ressort des données rassemblées ci-dessus. Il est clair que les premières sont, à cet égard, supérieures aux secondes, tant en ce qui concerne le gain de poids quotidien moyen que l'indice moyen de consommation. Ces résultats n'ont, néanmoins, de signification précise qu'en ce qui concerne les échantillons étudiés et ne sauraient être généralisés sans de nouveaux essais.

Dans le cadre des levures de betteraves, celle séchée par atomisation a permis d'enregistrer les gains de poids les plus élevés et celle ayant subi la dessiccation à haute température les moins bons, ce qui correspond sensiblement aux différences d'appétence enregistrées. Dans le cas des levures de mélasse, le lot séché à basse température s'est, par contre, montré le plus médiocre, et diverses explications peuvent être proposées. Il est possible que l'autolyse n'évolue pas identiquement dans les deux types de levure et qu'elle ne conduise à des processus réduisant la valeur alimentaire que dans le cas de l'une d'elles. Le chauffage peut, par contre, faire disparaître de celle-ci un produit gênant son utilisation (inhibiteur d'enzymes).

L'administration de levures à des taux aussi élevés ne permet pas de juger avec précision de leur valeur alimentaire, et ces essais n'ont de signification que sur le plan comparatif, car l'excès de certains constituants des levures dans les régimes est susceptible d'avoir entraîné des troubles sur lesquels notre attention n'a pas été attirée. C'est ainsi que les résultats médiocres obtenus lors de l'ingestion massive de levure de mélasse ne relèvent pas nécessairement que de la qualité des protéines de celle-ci.

De toute façon, si ces conclusions ne sauraient être généralisées avant d'avoir effectué une étude systématique portant sur un plus grand nombre d'échantillons, il paraît légitime d'attribuer, pour une large part, les différences observées à l'appétence diverse des animaux vis-à-vis des régimes. Celle-ci apparaît comme un facteur extrêmement important de la qualité d'un aliment. 


\section{BIBLIOGRAPHIE}

(I) Braude (R.), Kon (S. K.) et White (E. G.). - Yeast as a protein supplement for pigs and its relation to the appearance of rickets. J. Comp. Path. Ther. $53 ; 2,161,1943$.

(2) Braude (E.) et Kon (S. K.). - Yeast as a protein supplement for pigs : further observations on its rachitogenic effect. J. Comp. Path. Ther. 54; 2 ; 88, I944.

(3) Clauden $(\mathrm{H}$, ). - 39. Beretning om Sammenlignende forsg med Svin. 256. Beretning fra Forsoglaboratoriet, 195I.

(4) FÉvRJER (R.). - L'indice de consommation est-il le témoin fidèle de l'efficacité d'une ration? Annales de Zootechnie; 1 ; 175 -1952.

(5) Gartner (R.) et Gaede (V.). - Uber den Einfluss der Fütterung von bestrahlter Hefe auf das Wachstum von Mastschweinen. Landwirtsch. Jahrb. $75 ; 75$ I, 1932.

(6) LERoy (A. M.) et Février (R.). - Emploi de la levure de distillerie pour l'alimentation des animaux domestiques. Ann. Agron. $4 ; 578$, I947.

(7) LERoy (A. M.) et FéVRIER (R.). - Influence du mode de distribution des repas sur l'utilisation de la nourriture par le porc. Ann. Agron. 5 ; 769, I947.

(8) Leroy (A. M.) et FÉvrier (R.). - Nombre des repas et utilisation de la nourriture par le porc. Ann. Agron. 4 ; 605, I949.

(9) Leroy (A. M.) et FÉvrier (R.). - Étude des aptitudes économiques et de leur transmission chez les différentes souches et races porcines. Ann. Agron. 6 ; 925 , I949.

(io) Macrae (T. F.), El-Sadr (M. M.) et Sellers (K. C.). - The nutritive value of yeast protein : comparison of the supplementary values of yeast protein and casein for maize protein in the nutrition of the pig. Biochem. J. 36; $460,1942$.

(I I) National Research Council. Recommended Nutrient Allowances for Swine. Washington. Rpt. $\mathrm{n}^{\mathrm{O}} \mathrm{II}$.

(I2) Tosic (J.). - Effect of small quantities of a yeast preparation on the recovery of appetite in sheep. Brit. J. Nutrition, $3 ; 2-3 ; 234,1949$. 